\title{
BMJ Open Efficacy and safety of bevacizumab plus erlotinib versus bevacizumab or erlotinib alone in the treatment of non-small-cell lung cancer: a systematic review and meta-analysis
}

\author{
Shu Zhang, ${ }^{1}$ Xiao-dong Mao, ${ }^{2}$ Hai-tao Wang, ${ }^{1}$ Feng Cai, ${ }^{1}$ Jing $\mathrm{Xu}{ }^{1}$
}

To cite: Zhang S, Mao Xdong, Wang $\mathrm{H}$-tao, et al. Efficacy and safety of bevacizumab plus erlotinib versus bevacizumab or erlotinib alone in the treatment of non-small-cell lung cancer: a systematic review and meta-analysis. BMJ Open 2016;6:e011714. doi:10.1136/bmjopen-2016011714

- Prepublication history for this paper is available online. To view these files please visit the journal online (http://dx.doi.org/10.1136/ bmjopen-2016-011714).

SZ and X-dM contributed equally to this research.

Received 2 March 2016 Revised 26 May 2016 Accepted 27 May 2016 CrossMark

\footnotetext{
${ }^{1}$ Department of Respiratory Medicine, Seventh People's Hospital of Shanghai University of TCM, Shanghai, China

${ }^{2}$ Department of Chest Surgery, Seventh People's Hospital of Shanghai University of TCM, Shanghai, China
}

Correspondence to Dr Shu Zhang; zhangshu_2008@126.com

\section{ABSTRACT}

Objectives: Bevacizumab and erlotinib inhibit different tumour growth pathways, and both exhibit beneficial effects in the treatment of non-small-cell lung cancer (NSCLC). However, the efficacy of bevacizumab in combination with erlotinib remains controversial. Therefore, we conducted a meta-analysis to compare combination treatment with bevacizumab and erlotinib to bevacizumab or erlotinib monotherapy in the treatment of NSCLC.

Methods: Randomised controlled trials (RCTs) published in PubMed, Web of Science and EMBASE were systematically reviewed. The main outcome measures included overall survival (OS), progressionfree survival (PFS), overall response rate (ORR) and adverse events. Results were expressed as HRs or risk ratios (RRs) with $95 \%$ Cls.

Results: 5 RCTs involving a total of 1736 patients were included in this meta-analysis. The combination of bevacizumab and erlotinib significantly improved PFS ( $\mathrm{HR}=0.63,95 \% \mathrm{Cl} 0.53$ to $0.75 ; \mathrm{p}=0.000$ ) and the ORR ( $R R=1.91,95 \% \mathrm{Cl} 1.19$ to $3.06 ; p=0.007$ ) in the second-line treatment of NSCLC compared with bevacizumab or erlotinib alone. However, no significant difference in OS was observed between the combination and monotherapy groups ( $\mathrm{HR}=0.96,95 \% \mathrm{Cl} 0.83$ to $1.11 ; p=0.573$ ). A subgroup analysis has shown that the greatest PFS benefit was associated with an age of $<65$ years $(\mathrm{HR}=0.74,95 \% \mathrm{Cl} 0.57$ to $0.96 ; \mathrm{p}=0.026)$, Asian/Pacific Islander ethnicity (HR=0.23, 95\% Cl 0.10 to $0.54 ; p=0.001$ ), Eastern Cooperative Oncology Group performance status (ECOG PS) 1 (HR=0.82, $95 \% \mathrm{Cl}$ 0.68 to $0.98 ; p=0.033$ ), stage IIIB or IV disease (HR=0.68, 95\% $\mathrm{Cl} 0.57$ to $0.82 ; \mathrm{p}=0.000$ ) and no history of smoking (HR=0.48, $95 \% \mathrm{Cl} 0.32$ to 0.71 ; $p=0.000$ ). The incidence of grade $3 / 4$ adverse events such as rash and diarrhoea was higher in the combination group than in the monotherapy group.

Conclusions: The addition of bevacizumab to erlotinib can significantly improve PFS and the ORR in the second-line treatment of NSCLC with an acceptable and manageable risk of rash and diarrhoea. Further wellconducted, large-scale trials are needed to validate these findings.

\section{Strengths and limitations of this study}

- This is the first systematic review and meta-analysis of randomised controlled trials (RCTs) to compare combination treatment with bevacizumab and erlotinib to bevacizumab or erlotinib monotherapy in the treatment of non-small-cell lung cancer (NSCLC).

- Five RCTs involving a total of 1736 patients were identified. All these studies included were highquality, well-performed trials.

- We found that a combination of bevacizumab and erlotinib significantly improved progressionfree survival and the overall response rate in the second-line treatment of NSCLC compared with bevacizumab or erlotinib alone. However, no significant difference in OS was observed between the combination and monotherapy groups.

- Owing to the limited number of included studies and small sample size, the treatment effects of combination treatment with bevacizumab and erlotinib might be overestimated. Therefore, physicians should interpret our findings with caution when applying them in clinical practice.

\section{INTRODUCTION}

Lung cancer is the leading cause of cancerrelated death globally, ${ }^{1}{ }^{2}$ accounting for almost 1.2 million deaths annually. ${ }^{3}$ In particular, more than $85 \%$ of patients with lung cancer are diagnosed with non-small-cell lung cancer (NSCLC). ${ }^{1}$ Approximately $75 \%$ of patients diagnosed with NSCLC present with advanced disease. Although $30-40 \%$ of patients have a good response to cytotoxic therapy initially, all patients eventually experience progression during or after treatment. ${ }^{4}$

Bevacizumab (Bev) is a recombinant monoclonal antibody targeting the vascular endothelial growth factor (VEGF). ${ }^{5}$ It has been approved by the US Food and Drug Administration (FDA) for the first-line 
treatment of patients with unresectable, locally advanced or metastatic non-squamous NSCLC in combination with paclitaxel and carboplatin. ${ }^{5}$ A phase 3 study examined this combination in patients with NSCLC, ${ }^{6}$ finding that the regimen significantly improved overall survival (OS) and progression-free survival (PFS) in patients with NSCLC compared with the effects of paclitaxel and carboplatin. ${ }^{6}$ Another phase 3 study examining Bev plus cisplatin and gemcitabine in the first-line treatment of non-squamous NSCLC also uncovered the beneficial effects regarding PFS and the overall response rate (ORR), although the regimen failed to prolong OS. ${ }^{7}$

Erlotinib (Erl) is a small-molecule inhibitor targeting the epidermal growth factor receptor (EGFR). ${ }^{8}$ It has been approved by the US FDA for the treatment of locally advanced or metastatic NSCLC following the failure of one or more prior chemotherapy (CT) regimens. A phase 3 study has shown that OS was prolonged in patients with NSCLC when Erl was used in combination with second-line or third-line monotherapy. ${ }^{8}$ Furthermore, Erl is also approved by the US FDA for the first-line treatment of patients with metastatic NSCLC whose tumours have EGFR exon 19 deletions or exon 21 (L858R) substitution mutations. ${ }^{9}$

As Bev and Erl target different tumour growth pathways (VEGF and EGFR, respectively) with complementary mechanisms in the treatment of tumours, ${ }^{10-12}$ it is possible that their combined use would produce better effects than either agent alone. In a double-blind, placebo-controlled, phase 3 trial, this combination regimen significantly prolonged PFS in patients with recurrent or refractory NSCLC compared with the effects of Erl alone (median 3.4 vs 1.7 months; HR=0.62, 95\% CI 0.52 to 0.75$).{ }^{13}$ However, a contradictory result was obtained in another phase 2 study, in which the combination therapy shortened PFS compared with Bev +CT (BC; 18.4 vs25.0 months; HR=2.05, 95\% CI 1.11 to 3.77). ${ }^{14}$ The treatment line might affect the efficacy of Bev and Erl. We therefore conducted a meta-analysis based on relevant randomised controlled trials (RCTs) to assess the efficacy and safety of Bev+Erl (BE) in the first-line or second-line treatment of patients with NSCLC.

\section{MATERIALS AND METHODS \\ Literature search}

A comprehensive search was conducted to identify the relevant studies on the use of Bev and $\mathrm{Erl}$ in the treatment of NSCLC. PubMed, EMBASE, Web of Science and the Cochrane library were searched for all studies published before 18 January 2016. The search terms used were ('carcinoma, non-small-cell lung' [MeSH Terms] OR ('carcinoma' [All Fields] AND 'non-smallcell' [All Fields] AND 'lung' [All Fields]) OR 'non-smallcell lung carcinoma' [All Fields] OR ('non' [All Fields] AND 'small' [All Fields] AND 'cell' [All Fields] AND 'lung' [All Fields] AND 'cancer' [All Fields]) OR ('non small cell lung cancer' [All Fields]) AND ('bevacizumab' [Supplementary Concept] OR 'bevacizumab' [All Fields]) AND ('erlotinib' [Supplementary Concept] OR 'erlotinib' [All Fields]). We also manually searched the reference lists of the selected articles until no additional potential articles could be identified.

\section{Review strategy}

EndNote bibliographic software was used to create an electronic library of citations identified in the literature searches. PubMed, EMBASE, Web of Science and Cochrane library searches were performed using EndNote, and duplicate records were deleted. Two independent investigators ( $\mathrm{SZ}$ and $\mathrm{X}-\mathrm{dM}$ ) were trained to perform the abstract review and a full-text review thereafter. Disagreements between the investigators were resolved by consensus and discussion.

\section{Study inclusion and exclusion criteria}

All clinical trials comparing the efficacy and safety of BE with other agents in the treatment of NSCLC were considered eligible for the analysis. The inclusion criteria were as follows: (1) RCTs; (2) the patients were $\geq 18$ years of age and had histologically or cytologically confirmed NSCLC; (3) patients were randomly assigned to receive $\mathrm{BE}$ or $\mathrm{Bev} / \mathrm{Erl}$ alone; (4) data were presented for OS, PFS, the ORR (complete and partial responses) and the incidence of adverse events; (5) the full text was available. Reviews, comments, case report, editorials, letters or articles unrelated with our topics were excluded from the final analysis.

\section{Data extraction and quality assessment}

Data were extracted from the selected studies independently by two investigators ( $\mathrm{SZ}$ and $\mathrm{X}-\mathrm{dM}$ ) using a standardised data extraction method. The following data were extracted: first author, numbers of patients in the study and control groups, baseline patient characteristics, HRs with $95 \%$ CIs for OS and PFS, and incidence of major adverse events (eg, hypertension, rash, haemorrhage, diarrhoea, proteinuria and interstitial lung disease-like events).

Two investigators (H-tW and FC) independently assessed the risk of bias in included RCTs with the method recommended by the Cochrane Collaboration. ${ }^{15}$ The quality of evidence for the outcome measures was evaluated using the Grading of Recommendations Assessment, Development and Evaluation (GRADE) approach. $^{16}$

\section{Statistical analysis}

Analyses were performed in the intent-to-treat population. We first tested the heterogeneity between the studies using $\mathrm{I}^{2}$ statistics. ${ }^{17}$ The studies were considered to have low, moderate or high heterogeneity when the value of $\mathrm{I}^{2}$ was $25-50 \%, 50-75 \%$ or $>75 \%$, respectively. ${ }^{18}$ 
An $\mathrm{I}^{2}$ value $>50 \%$ indicates significant heterogeneity. When heterogeneity was found among the included studies, a random-effects model ${ }^{19}$ was used to pool the estimates; otherwise, a fixed-effects model was applied. ${ }^{20}$ Time-to-event variables, including OS and PFS, were expressed as HRs with 95\% CIs for each study. Dichotomous variables, including the ORR and incidence of adverse events, were expressed as risk ratios (RRs) with 95\% CIs for each study. In some studies, Kaplan-Meier curves were provided instead of HR and 95\% CI; in these cases, we used the method described by Tierney et $a l^{21}$ to estimate the HR and 95\% CI from the Kaplan-Meier curves. Subgroup analysis was conducted on the basis of the line of treatment and comparators. We also explored the treatment effects in subpopulations of patients defined by demographics and baseline characteristics, including age, race, Eastern Cooperative Oncology Group performance status (ECOG PS), disease stage and smoking history. Since the number of included studies was $<10$, publication bias was not assessed. A $p<0.05$ was considered statistically significant. All analyses were performed using STATA V.12.0 (Stata Corporation, College Station, Texas, USA).

Figure 1 Search strategy and flow chart for this meta-analysis.

\section{RESULTS}

\section{Identification of eligible studies}

The initial search yielded 723 records from PubMed, EMBASE, Web of Science and the Cochrane library. Of these, 438 studies were removed as duplicate records, and 228 studies were excluded after a review of the title/abstract (figure 1). Then, 57 potential studies were identified for the full-text review. Among them, 42 studies unrelated with our topics were excluded for the following reasons: 19 were not targeted population (patients $<18$ years old or with small-cell lung cancer); 23 were not targeted interventions (Bev combined with other agents rather than Erl). Additionally, another10 studies were excluded for the following reasons: two were study design (trial protocol), ${ }^{22}{ }^{23}$ six studies were single-arm trials ${ }^{24-29}$ and two studies did not provide outcomes of interest. ${ }^{30}{ }^{31}$ Finally, five RCTs (involving 1736 patients) ${ }^{13} \quad 14 \quad 32-34$ met the inclusion criteria, and they were included in this meta-analysis.

Characteristics of eligible studies and quality assessment The main characteristics of all eligible RCTs are presented in table 1 . These studies were published between 2007 and 2014. The sample size of the studies ranged 
Table 1 Baseline characteristics of the patients in the trials included in the meta-analysis

\begin{tabular}{|c|c|c|c|c|c|c|c|}
\hline Author & Treatment regimen & $\begin{array}{l}\text { Number of } \\
\text { patients }\end{array}$ & $\begin{array}{l}\text { Male/ } \\
\text { female }\end{array}$ & $\begin{array}{l}\text { Smoking history } \\
\text { (never/previous/ } \\
\text { current) }\end{array}$ & $\begin{array}{l}\text { ECOG } \\
\text { PS(0/1/2) }\end{array}$ & $\begin{array}{l}\text { Histology (large-cell } \\
\text { carcinoma/adenocarcinoma/ } \\
\text { squamous/others) }\end{array}$ & $\begin{array}{l}\text { Line of } \\
\text { treatment }\end{array}$ \\
\hline \multirow[t]{2}{*}{ Herbst et al ${ }^{13}$} & $\begin{array}{l}\text { Bev } 15 \text { mg/kg(3-week cycle)+erlo } \\
150 \text { mg per day }\end{array}$ & 319 & $171 / 148$ & $34 / 237 / 48$ & $129 / 166 / 23$ & $23 / 242 / 11 / 43 / 38$ & Second-line \\
\hline & Erlo $150 \mathrm{mg}$ per day+placebo $15 \mathrm{mg} / \mathrm{kg}$ & 317 & $170 / 147$ & $33 / 212 / 72$ & $121 / 176 / 20$ & $25 / 235 / 17 / 40$ & \\
\hline \multirow[t]{2}{*}{ Ciuleanu et $a l^{14}$} & $\begin{array}{l}\text { Bev } 15 \mathrm{mg} / \mathrm{kg} \text { (3-week cycle)+erlo } \\
150 \mathrm{mg} \text { per day }\end{array}$ & 63 & $37 / 26$ & $21 / 20 / 11$ & $28 / 35 / 0$ & NR & First-line \\
\hline & $\begin{array}{l}\text { Bev15 mg/kg (3-week cycle) } \\
+(\text { gemcitabine/cisplatin or carboplatin/ } \\
\text { paclitaxel) }\end{array}$ & 61 & $36 / 25$ & $23 / 14 / 24$ & $20 / 41 / 0$ & NR & \\
\hline \multirow[t]{2}{*}{ Seto et $a 1^{\beta 2}$} & Bev 15 mg/kg+erlo 150 mg per day & 77 & $30 / 45$ & $42 / 9 / 24$ & $43 / 32 / 0$ & $0 / 74 / 1 / 0$ & First-line \\
\hline & Erlo 150 mg per day & 77 & $26 / 51$ & $45 / 6 / 26$ & $41 / 36 / 0$ & $1 / 76 / 0 / 0$ & \\
\hline \multirow[t]{2}{*}{ Herbst et $a l^{\beta 3}$} & $\begin{array}{l}\text { Bev } 15 \mathrm{mg} / \mathrm{kg} \text { (3-week cycle)+erlo } \\
150 \mathrm{mg} \text { per day }\end{array}$ & 39 & $17 / 22$ & NR & $19 / 20 / 0$ & $0 / 32 / 7 / 0$ & Second-line \\
\hline & $\begin{array}{l}\text { Bev } 15 \mathrm{mg} / \mathrm{kg} \text { (3-week cycle)+chemo } \\
\text { (docetaxel or pemtrexed) }\end{array}$ & 40 & $23 / 17$ & NR & $19 / 21 / 0$ & $9 / 30 / 1 / 0$ & \\
\hline \multirow[t]{2}{*}{ Johnson et $a \AA^{\beta 4}$} & $\begin{array}{l}\text { Bev } 15 \text { mg/kg(3-week cycle)+erlo } \\
150 \text { mg per day }\end{array}$ & 370 & $193 / 177$ & $61 / 180 / 129$ & $180 / 190 / 0$ & $30 / 301 / 11 / 28$ & Second-line \\
\hline & Bev15 mg/kg(3-week cycle)+placebo & 373 & $196 / 177$ & $66 / 178 / 129$ & $173 / 198 / 1$ & $26 / 309 / 6 / 32$ & \\
\hline
\end{tabular}

Bev, bevacizumab; erlo, erlotinib; ECOG PS, Eastern Cooperative Oncology Group performance status; NR, not reported. 
from 79 to 743 . Among the included studies, three were conducted in the USA, ${ }^{13} 33{ }^{34}$ one in $\mathrm{Japan}^{32}$ and another one was conducted in multiple countries, ${ }^{14}$ including Romania, Taiwan, Poland, Italy, Korea, the Netherlands, Switzerland and the UK. Three studies assessed the combination of Bev and Erl as second-line therapy, ${ }^{13} 3334$ and two studies evaluated the combination as first-line therapy. ${ }^{142}$ Two studies compared the combination of Bev and Erl with Erl alone, ${ }^{13} 32$ and three studies compared the combination regimen with Bev alone. ${ }^{1433} 34$ The ATLAS study was initially reported at the 2009 ASCO annual meeting by Miller et $a l^{35}$ updated in 2010 by Kabbinavar et $a l^{36}$ and finally presented in 2013 by Johnson et $a l^{34}$ We included the latest version of the study published in $2013^{34}$ and excluded the original and updated versions.

The details of the risk-of-bias assessment are summarised in figure 2. Two trials were judged to be at low risk of bias and three at unclear risk of bias. Five trials generated an adequate randomisation sequence and appropriate allocation concealment. The GRADE level of evidence was low for OS, PFS and ORR.

\section{Overall survival}

All of the studies reported OS data. ${ }^{13} 14{ }^{32-34}$ The OS in the combination and monotherapy groups were 9.3 and

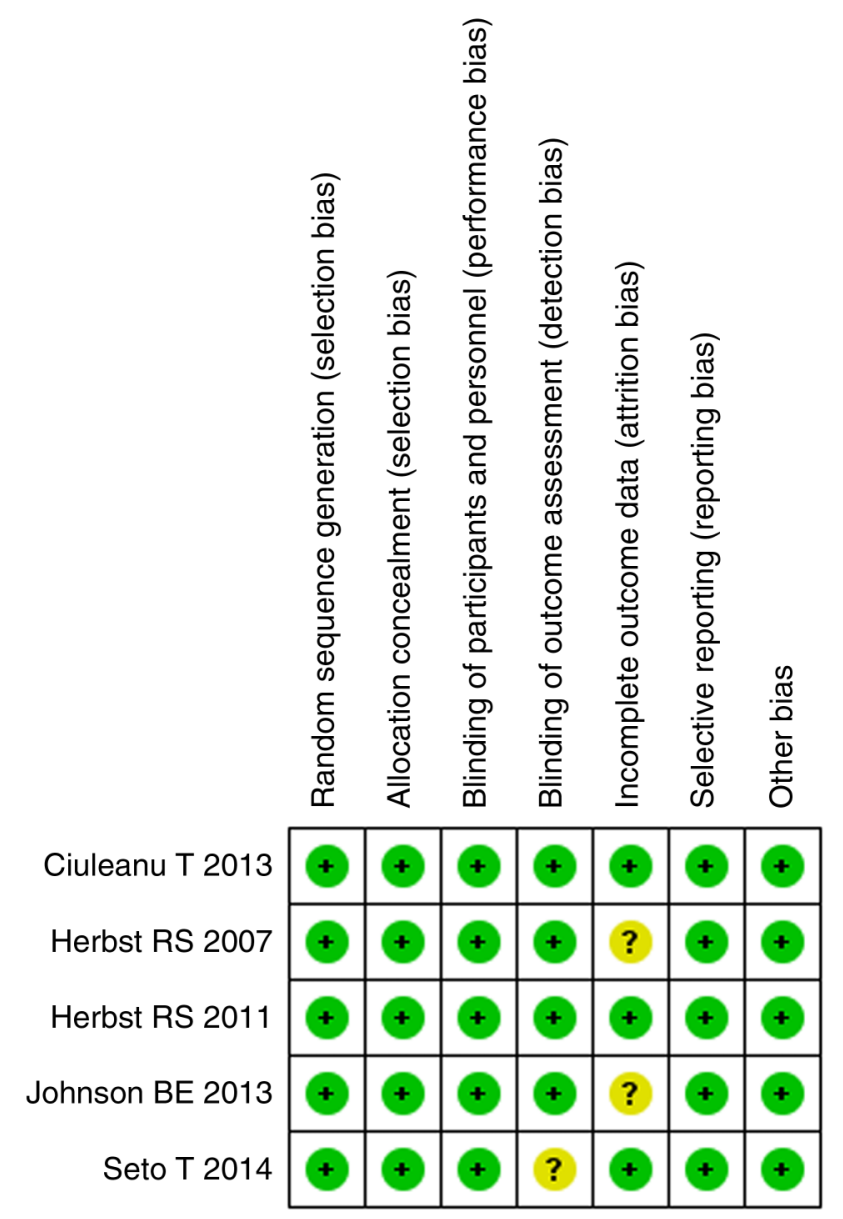

Figure 2 Risk of bias summary.
9.2 months $(\mathrm{p}=0.758), 16.4$ and 16.1 months $(\mathrm{p}=0.406)$, 13.9 and 12.8 months $(\mathrm{p}=0.165), 13.7$ and 12.6 months $(p>0.05)$, and 14.4 and 13.3 months $(p=0.534)$, respectively, which indicated that combination treatment did not prolong the survival time of patients with NSCLC. The pooled estimates suggested that the combined use of Bev and Erl was not associated with any significant improvement in OS $(\mathrm{HR}=0.96,95 \%$ CI 0.83 to 1.11; $\mathrm{p}=0.573$; figure 3$)$. No heterogeneity was identified among the included studies $\left(\mathrm{p}=0.748, \mathrm{I}^{2}=0.0 \%\right)$.

We also performed subgroup analysis based on the line of treatment and comparators. The aggregated results illustrated that $\mathrm{BE}$ did not prolong OS in patients with NSCLC when used as first-line ( $\mathrm{HR}=0.98,95 \%$ CI 0.78 to $1.24 ; \mathrm{p}=0.895)$ or second-line therapy $(\mathrm{HR}=0.94$, $95 \%$ CI 0.79 to $1.13 ; \mathrm{p}=0.541$ ). Combination therapy did not improve OS when it was compared with either Bev $(\mathrm{HR}=0.94,95 \%$ CI 0.76 to $1.17 ; \mathrm{p}=0.602)$ or $\mathrm{Erl}$ $(\mathrm{HR}=0.97,95 \%$ CI 0.80 to $1.18 ; \mathrm{p}=0.770)$.

\section{Progression-free survival}

All of the studies presented data for PFS..$^{13} 14$ 32-34 The PFS in the combination and monotherapy groups were 3.4 and 1.7 months $(p<0.001), 25.0$ and 18.4 weeks $(p=0.018), 16.0$ and 9.7 months $(\mathrm{p}=0.0015), 4.4$ and 4.8 months $(\mathrm{p}>0.05)$, and 4.8 and 3.7 months $(\mathrm{p}<0.001)$, respectively, which indicated that combination treatment could prolong the progression-free time of patients with NSCLC. The pooled results indicated that the combination of Bev with Erl significantly improved PFS compared with Bev or Erl alone ( $\mathrm{HR}=0.74,95 \%$ CI 0.56 to $0.98 ; \mathrm{p}=0.035$; figure 4 ). The test for heterogeneity was significant $\left(\mathrm{p}=0.005, \mathrm{I}^{2}=73.4 \%\right)$. Therefore, we conducted sensitivity analysis to explore the potential source of heterogeneity. The exclusion of one study conducted by Ciuleanu et $a l^{14}$ yielded similar results ( $\mathrm{HR}=0.65,95 \%$ CI 0.57 to $0.73 ; \mathrm{p}=0.000$ ), but no evidence of heterogeneity was found among the remaining studies $\left(\mathrm{p}=0.582, \mathrm{I}^{2}=0.0 \%\right)$. When we excluded the other four studies individually, the overall estimates and heterogeneity did not change substantially.

We also performed a subgroup analysis based on the line of treatment and comparators. The aggregated results demonstrated that Bev in combination with Erl prolonged PFS in the second-line treatment of patients with NSCLC (HR=0.63, 95\% CI 0.53 to $0.75 ; \mathrm{p}=0.000)$, but this effect was not observed for first-line therapy ( $\mathrm{HR}=0.86,95 \%$ CI 0.49 to $1.52 ; \mathrm{p}=0.614)$. A combination of Bev with Erl significantly improved PFS compared with either Bev $(\mathrm{HR}=0.60,95 \%$ CI 0.51 to $0.71 ; \mathrm{p}<0.001)$ or Erl alone (HR=0.78, 95\% CI 0.65 to $0.93 ; \mathrm{p}=0.006)$.

\section{PFS in subpopulations of patients}

Four studies reported PFS data for subpopulations of patients. ${ }^{13} \quad 14 \quad 32 \quad 34$ The aggregated results of these studies suggested that the combined therapy was associated with prolonged PFS in patients who were $<65$ years of age $(\mathrm{HR}=0.74,95 \%$ CI 0.57 to 0.96 ; $\mathrm{p}=0.026)$ but not in those older than 65 years $(\mathrm{HR}=0.71$, 


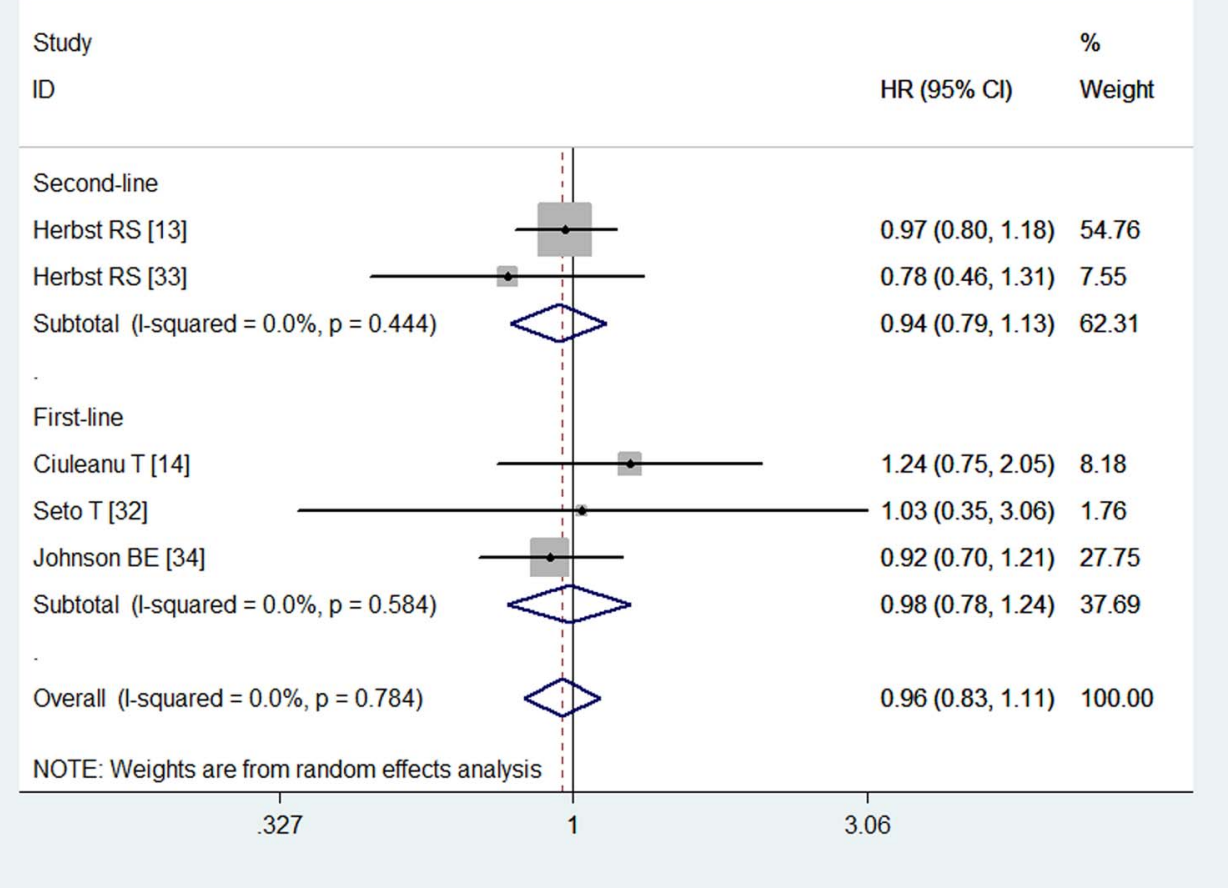

Figure 3 Overall survival for combination therapy of bevacizumab plus erlotinib with bevacizumab or erlotinib alone.

95\% CI 0.46 to $1.12 ; \mathrm{p}=0.140)$. Regarding ethnicity, the combined therapy was associated with prolonged PFS in Asians or Pacific Islanders $(\mathrm{HR}=0.23,95 \%$ CI 0.10 to $0.54 ; \mathrm{p}=0.001)$ but not in white patients $(\mathrm{HR}=0.87,95 \%$ CI 0.65 to $1.18 ; \mathrm{p}=0.382$ ). Concerning ECOG PS, the combined therapy was associated with prolonged PFS in patients with ECOG PS1 (HR=0.82, 95\% CI 0.68 to 0.98 ; $\mathrm{p}=0.033$ ) but not those with ECOG PS0 (HR=0.72, 95\%
CI 0.48 to $1.07 ; \mathrm{p}=0.100$ ). Regarding smoking history, the combined therapy was associated with prolonged PFS in patients with no history of smoking ( $\mathrm{HR}=0.48$, $95 \%$ CI 0.32 to $0.71 ; \mathrm{p}=0.000$ ), but not in patients who currently or previously smoked (HR=0.77, 95\% CI 0.51 to $1.16 ; \mathrm{p}=0.209$ ). Concerning disease stage, the combined therapy was associated with prolonged PFS in patients with stage IIIB or IV disease ( $\mathrm{HR}=0.68,95 \%$ CI

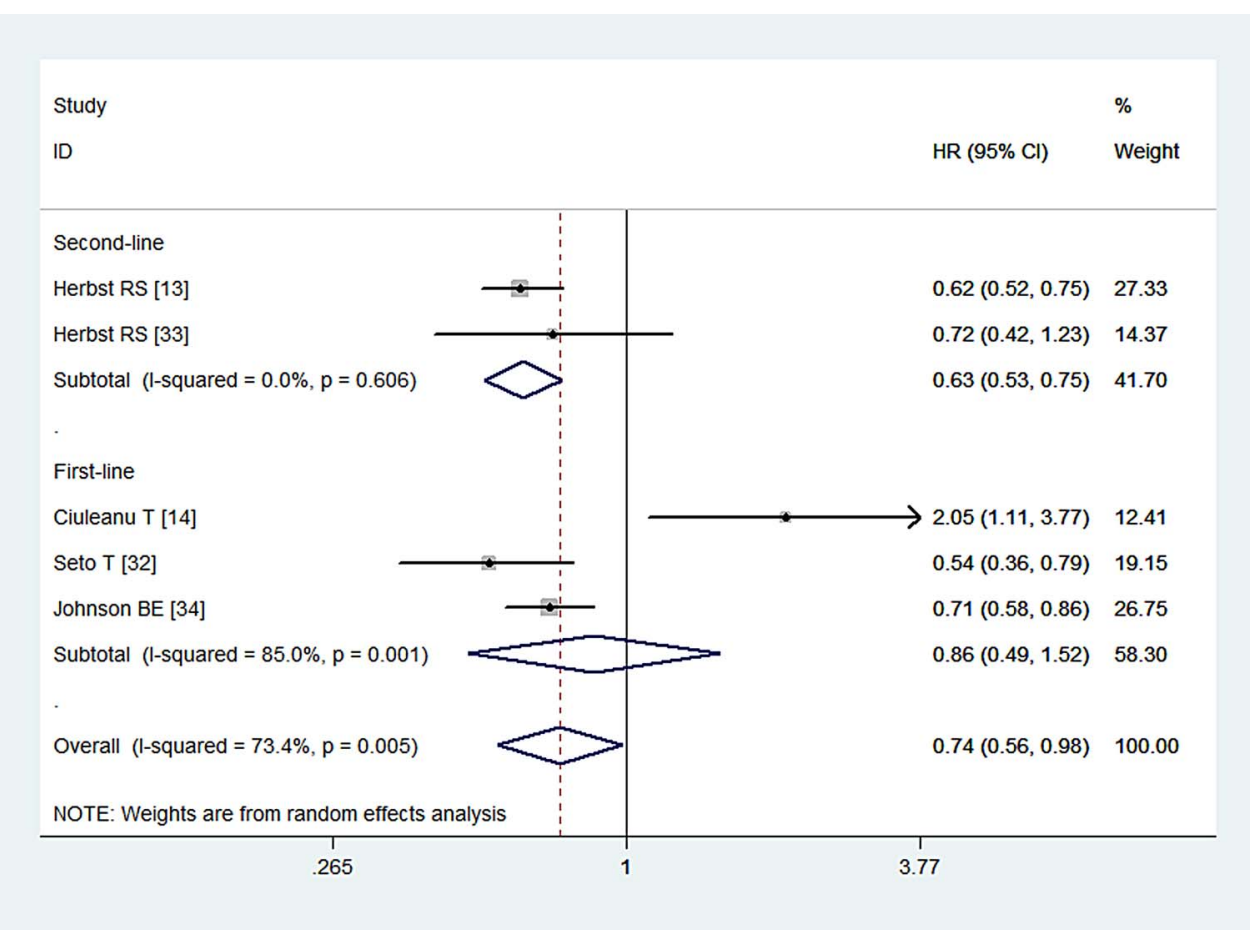

Figure 4 Progression-free survival for combination therapy of bevacizumab plus erlotinib with bevacizumab or erlotinib alone. 
0.57 to $0.82 ; \mathrm{p}=0.000)$, but not in those with recurrent disease $(\mathrm{HR}=0.46,95 \%$ CI 0.16 to $1.34 ; \mathrm{p}=0.154)$.

\section{Overall response rate}

Four studies reported ORR data. ${ }^{13} 143233$ The ORR in the combination and monotherapy groups was $12.6 \%$ and $6.2 \%, 69.3 \%$ and $63.6 \%, 23.8 \%$ and $34.4 \%, 17.9 \%$ and $12.2 \%$, respectively. The pooled results indicated that patients with NSCLC who were treated with combination therapy had similar ORRs as those treated with Bev or Erl alone ( $R R=1.19,95 \%$ CI 0.77 to $1.83 ; \mathrm{Z}=2.72$, $\mathrm{p}=0.438$; figure 5). We also performed subgroup analysis based on the line of treatment and comparators. The aggregated results illustrated that second-line treatment with $\mathrm{BE}$ increased the ORR in patients with NSCLC $(\mathrm{RR}=1.91$, 95\% CI 1.19 to 3.06; $\mathrm{p}=0.007)$, but this effect was not observed for first-line treatment with this regimen (RR=0.93, 95\% CI 0.59 to $1.46 ; \mathrm{p}=0.747$ ). Patients with NSCLC who were treated with combination therapy had similar ORRs as those treated with either Bev ( $R R=1.43,95 \%$ CI 0.72 to $2.85 ; \mathrm{p}=0.304)$ or $\mathrm{Erl}$ alone $(\mathrm{RR}=0.88,95 \%$ CI 0.44 to $1.75 ; \mathrm{p}=0.713)$.

\section{Adverse events}

All of the included studies reported the data of adverse events. $^{13} 14 \quad 32-34$ The most common adverse events of grade $3 / 4$ are listed in table 2 . The pooled estimates suggested that the combination therapy of Bev and Erl induced a significantly higher rate of rash $(R R=2.59$, 95\% CI 1.40 to $4.79 ; \mathrm{p}=0.002)$ and diarrhoea $(\mathrm{RR}=2.73$, 95\% CI 1.12 to $6.67 ; \mathrm{p}=0.027$ ) as compared with $\mathrm{Bev}$ or Erl alone.

\section{DISCUSSION}

The present study was a systematic review and meta-analysis of RCTs with the objective of comparing the combination of Bev and Erl to Bev or Erl alone in the treatment on NSCLC. Our study demonstrated that the combination treatment significantly prolonged PFS and elevated the ORR in the second-line treatment of NSCLC. Bev combined with Erl did not prolong OS in the treatment of NSCLC in either the first or the second line. In the subgroup analysis based on comparators, combination therapy significantly improved PFS and ORR, but not OS in the treatment of NSCLC when compared with either Bev or Erl alone. In addition, the addition of Bev to Erl significantly improved PFS in subpopulations of $<65$ years of age, Asians/Pacific Islanders, patients with ECOG 1, those with stage IIIB or IV disease and never-smokers. A higher incidence of grade 3 or 4 rash and diarrhoea was observed in the BE group. Our study indicated that patients with NSCLC could benefit from combined treatment with Bev and Erl.

There has been one published network meta-analysis of targeted drugs for unselected patients with advanced NSCLC. ${ }^{37}$ In that study, the authors included 24 RCTs to assess the efficacy and safety of targeted drugs and CT. They found that BC had a statistically significantly higher incidence of ORR relative to the other six different treatments, including Erl, gefitinib, cetuximab, CT, Erl+CT and placebo. ${ }^{37}$ These results were consistent with ours, in which combined therapy of Bev with Erl significantly improved the ORR in patients with NSCLC. Furthermore, in terms of the safety outcomes, both the $\mathrm{Bev}+\mathrm{CT}$ and Erl+CT were associated with higher

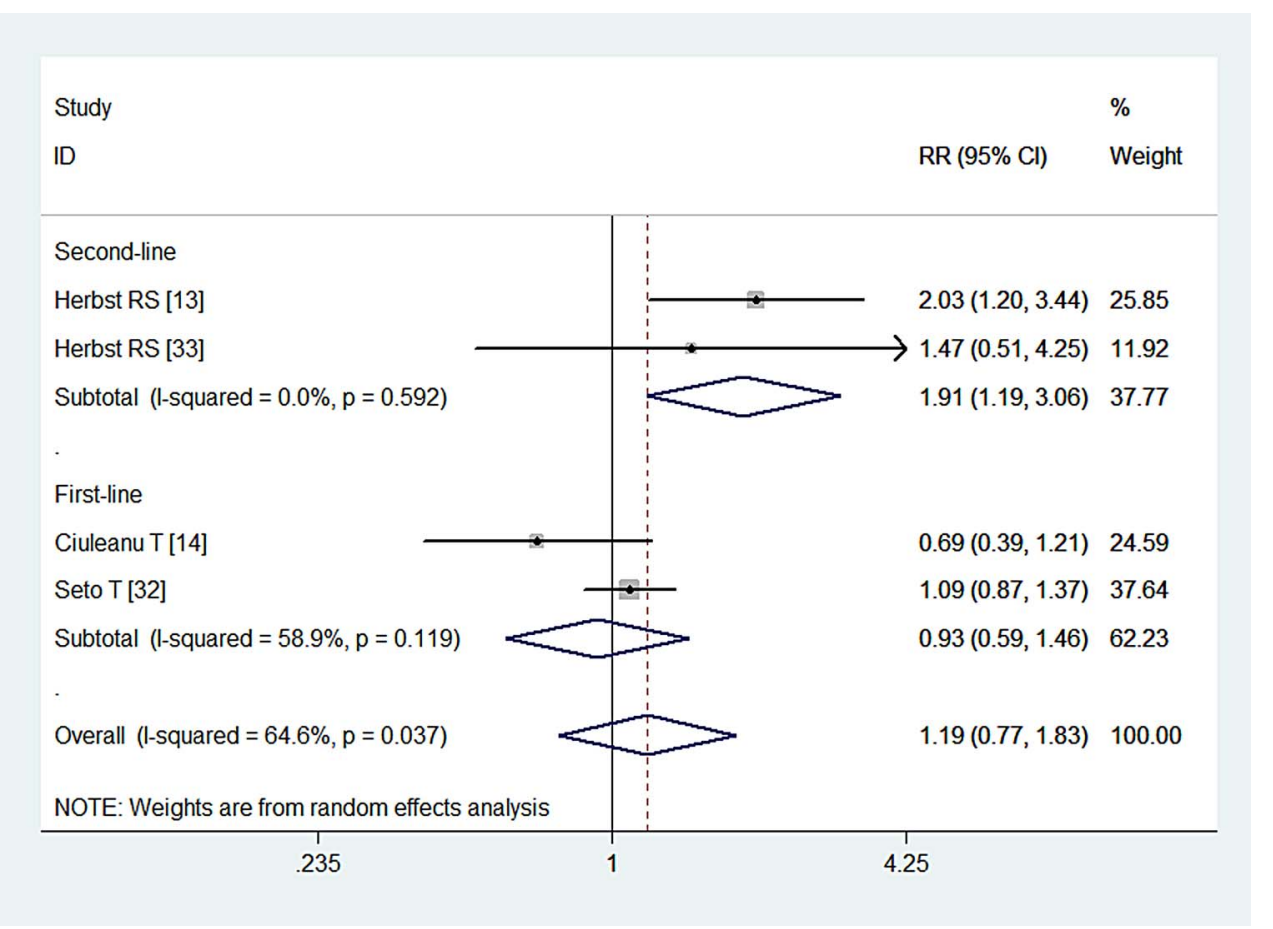

Figure 5 Overall response rate for combination therapy of bevacizumab plus erlotinib with bevacizumab or erlotinib alone. 
Table 2 Summary of the RRs of adverse events in patients with non-small-cell lung cancer

\begin{tabular}{lccccc}
\hline Adverse events & Incidence rate (comb group) & Incidence rate (mono group) & RR & 95\% Cl & p Value \\
\hline Hypertension & $84 / 795(10.57 \%)$ & $36 / 796(4.52 \%)$ & 2.19 & 0.73 to 6.61 & 0.164 \\
Rash & $144 / 933(15.43 \%)$ & $57 / 934(6.10 \%)$ & 2.59 & 1.40 to 4.79 & 0.002 \\
Haemorrhage & $18 / 756(2.38 \%)$ & $14 / 757(1.85 \%)$ & 1.28 & 0.65 to 2.52 & 0.480 \\
Proteinuria & $17 / 505(3.37 \%)$ & $9 / 505(1.78 \%)$ & 1.84 & 0.84 to 4.00 & 0.126 \\
Diarrhoea & $60 / 545(11.0 \%)$ & $20 / 544(3.68 \%)$ & 2.73 & 1.12 to 6.67 & 0.027 \\
ILD-like events & $4 / 681(0.59 \%)$ & $1 / 680(0.15 \%)$ & 3.00 & 0.47 to 18.96 & 0.244 \\
\hline Comb, combination; ILD-like events, interstitial lung disease-like events; mono, monotherapy; RR, risk ratio.
\end{tabular}

incidence of rash and diarrhoea than CT alone ${ }^{37}$ which was also observed in our study. In addition to these similar outcomes, we also explore the effects of combined therapy in PFS and OS, as well as the effects in different lines of treatments and comparators, which was not investigated in that network meta-analysis.

VEGF and EGFR play important roles in the growth and metastatic potential of cancers. Therefore, they have become therapeutic targets in the treatment of NSCLC. Previous phase $1 / 2$ trials presented strong biological evidence for the use of combined therapies that block these pathways. ${ }^{38-40}$ The JO25567 trial $^{32}$ was a randomised, multicentre, phase 2 study that compared the efficacy and safety of BE with those of Erl alone in patients with EGFR-mutant non-squamous NSCLC. The combination treatment significantly prolonged PFS (16.0 vs 9.7 months; HR $=0.72,95 \%$ CI 0.36 to 0.79 ; $\mathrm{p}=0.0015)$. This survival benefit of the combined therapy was also observed in the BeTa trial. ${ }^{13}$ In this trial, the addition of Bev to Erl significantly improved PFS (3.4 vs 1.7 months; HR=0.62, 95\% CI 0.52 to 0.75 ) and elevated the disease control rate $(45 \%$ vs $34 \%)$ compared with Erl alone. However, there was no significant difference in OS between the two groups $(9.3$ vs 9.2 months; HR $=0.97$, $95 \%$ CI 0.80 to $1.18 ; \mathrm{p}=0.758$ ). In the BeTa trial ${ }^{13} 355(56 \%)$ patients were screened for EGFR mutations, and only 30 were positive (12 in the combination group and 18 in the Erl group). Although the subgroup analysis data indicated a benefit in favour of patients with mutant EGFR compared with those with wild-type EGFR, the difference did not reach significance $(\mathrm{p}=0.1826)$.

An improvement of PFS associated with a combination of Bev and Erl was not observed in a multicentre, randomised phase 2 trial, ${ }^{33}$ in which BE was used as a secondline treatment for patients with recurrent or refractory non-squamous NSCLC. In this trial, 39 and 41 patients were randomly allocated to receive $\mathrm{BE}$ and $\mathrm{BC}$, respectively. The median PFS times for the $\mathrm{BE}$ and $\mathrm{BC}$ arms were 4.4 and 4.8 months (HR $=0.72,95 \%$ CI 0.42 to 1.23 ), respectively. The median $\mathrm{OS}$ times were 13.7 and 12.6 months for the $\mathrm{BE}$ and $\mathrm{BC}$ arms ( $\mathrm{HR}=0.78,95 \% \mathrm{CI}$ 0.46 to 1.31 ), respectively. The authors concluded that Bev in combination with Erl appeared to have similar efficacy as the combination of Bev and CT. These results might be attributable to the planned sample size.
Additionally, in another open-label, multicentre, randomised phase 2 trial, ${ }^{34}$ the combination of Bev and Erl resulted in adverse results. In this trial, 124 patients were enrolled (BE, $n=63 ; B C, n=61)$, but all of them had withdrawn from trial treatment by the time of the final analysis. Thus, the results were calculated from the updated interim analysis, in which only $30(47.6 \%)$ and 16 events $(26.2 \%)$ were observed in the $\mathrm{BE}$ and $\mathrm{BC}$ group, respectively. The results have shown that the median PFS times were 18.4 and 25.0 weeks for the $\mathrm{BE}$ and $\mathrm{BC}$ arms, respectively. The HR for PFS was 2.05 (95\% CI 1.11 to 3.77; $\mathrm{p}=0.0183$ ), which indicated a significant difference in favour of BC. The risk of death was higher in the $\mathrm{BE}$ arm $(19 \%)$ than in the BC arm $(11.5 \%)$, although the difference was not significant (HR=1.63, 95\% CI 0.64 to 4.15; $\mathrm{p}=0.2994)$. This updated analysis showed that the $\mathrm{BE}$ regimen produced a shorter PFS and a higher incidence of death than BC. The authors attributed these negative results to the small valid sample size in this study.

Regarding the efficacy of Bev and Erl as maintenance therapy, several studies have shown their beneficial effects. In a randomised, double-blind, phase IIIB trial, the addition of Erl to maintenance Bev significantly improved PFS in patients with advanced NSCLC. ${ }^{34}$ The mean PFS times for these two groups were 4.8 and 3.7 months, respectively. These results are comparable to data from some other studies illustrating a significant PFS benefit after maintenance therapy. In the AVAiL trial, the addition of Bev to platinum-based doublet CT significantly improved PFS compared with placebo in the treatment of advanced non-squamous NSCLC (6.5-6.7 vs 6.1 months). ${ }^{7}$ The Sequential Tarceva in Unresectable NSCLC study also reported significantly longer PFS and OS for patients who received maintenance Erl than for those who received placebo (mean PFS, 12.3 vs 11.1 weeks; mean OS, 12.0 vs 11.0 months). ${ }^{4142}$

Our study found no significant difference in the ORR between the BE and Bev or Erl monotherapy groups. This result was similar to those of three previous studies. ${ }^{32-34}$ Seto $e t a l^{22}$ noted that more patients in the combination group experienced a $>30 \%$ reduction in tumour size from baseline than in the Erl group, although the difference did not achieve statistical significance. These findings indicated that the combination of Bev and Erl might continue to exert a tumour-inhibiting 
effect after a reduction in tumour size is achieved, which might explain the PFS benefit associated with the combination treatment.

In this meta-analysis, we found that the incidence of grade 3 and 4 toxicities was almost similar between the combination and monotherapy groups, excluding that of rash and diarrhoea, which were more frequent in the combination treatment group. Although BE induced significantly higher rates of rash and diarrhoea than Bev or Erl alone, one trial ${ }^{33}$ found that the overall safety analysis favoured the combination regimen. Herbst $e t a l^{33}$ reported that only $13 \%$ of patients in the BE group discontinued treatment because of adverse events, compared with $28 \%$ of patients in the Bev alone group.

This meta-analysis had some potential limitations that should be considered. First, our study included only five RCTs, and some of these trials had relatively small sample sizes. Although all of these studies were highquality, well-performed trials, our conclusions should be interpreted with caution because smaller trials are more likely to result in overestimation of the treatment effect than larger trials. Second, our exploration of the effect of Bev in combination with Erl in patients with NSCLC with EGFR mutations was insufficient because of sparse reporting among the included studies. Finally, it should be noted that all of these trials were partly funded by the pharmaceutical industry, and their results might have been affected by the inherent conflict of interest and possible bias. However, these trials were all highquality studies that were conducted well, and they were the only eligible studies that evaluated the efficacy of the combination treatment. Therefore, physicians should interpret our findings with caution when applying them in clinical practice.

In conclusion, our meta-analysis indicated that the combination of Bev and Erl can significantly improve PFS and the ORR in the second-line treatment of nonsquamous NSCLC, but it did not prolong OS. Moreover, the combination treatment also produced an acceptable and tolerable risk of rash and diarrhoea. However, considering the potential limitations of this study, further large-scale trials are needed to verify our findings and explore the efficacy of the combined therapy in patients with non-squamous NSCLC with EGFR mutations.

Contributors SZ and X-dM conceived the study aims and design and contributed to the systematic review and data extraction. H-tW, FC and JX performed the analysis, interpreted the results and drafted the manuscript. All the authors contributed to the revision of data extraction, interpretation of results and to the review of the manuscript.

Funding This research received no specific grant from any funding agency in the public, commercial or from not-for-profit sectors.

\section{Competing interests None declared.}

Provenance and peer review Not commissioned; externally peer reviewed.

Data sharing statement Additional data can be accessed via the Dryad data repository at http://datadryad.org/ with the doi:10.5061/dryad.hb34s.

Open Access This is an Open Access article distributed in accordance with the Creative Commons Attribution Non Commercial (CC BY-NC 4.0) license, which permits others to distribute, remix, adapt, build upon this work noncommercially, and license their derivative works on different terms, provided the original work is properly cited and the use is non-commercial. See: http:// creativecommons.org/licenses/by-nc/4.0/

\section{REFERENCES}

1. Jemal A, Bray F, Center MM, et al. Global cancer statistics. CA Cancer J Clin 2011;61:69-90.

2. Jemal A, Siegel R, Xu J, et al. Cancer statistics, 2010. CA Cancer J Clin 2010;60:277-300.

3. Parkin DM, Bray F, Ferlay J, et al. Global cancer statistics, 2002. CA Cancer J Clin 2005;55:74-108.

4. Sakurada A, Shepherd FA, Tsao MS. Epidermal growth factor receptor tyrosine kinase inhibitors in lung cancer: impact of primary or secondary mutations. Clin Lung Cancer 2006;7(Suppl 4):S138-44.

5. Genentech. Avastin (bevacizumab): full prescribing information. South San Francisco, CA: Genentech, 2006.

6. Sandler A, Gray R, Perry MC, et al. Paclitaxel-carboplatin alone or with bevacizumab for non-small-cell lung cancer. $N$ Engl J Med 2006;355:2542-50.

7. Reck M, von Pawel J, Zatloukal P, et al. Phase III trial of cisplatin plus gemcitabine with either placebo or bevacizumab as first-line therapy for nonsquamous non-small-cell lung cancer: AVAil. J Clin Oncol 2009;27:1227-34.

8. Shepherd FA, Rodrigues Pereira J, Ciuleanu T, et al. Erlotinib in previously treated non-small-cell lung cancer. $N$ Engl J Med 2005;353:123-32.

9. Khozin S, Blumenthal GM, Jiang X, et al. U.S. Food and Drug Administration approval summary: erlotinib for the first-line treatment of metastatic non-small cell lung cancer with epidermal growth factor receptor exon 19 deletions or exon 21 (L858R) substitution mutations. Oncologist 2014;19:774-9.

10. Ciardiello F, Bianco R, Damiano V, et al. Antiangiogenic and antitumor activity of anti-epidermal growth factor receptor C225 monoclonal antibody in combination with vascular endothelial growth factor antisense oligonucleotide in human GEO colon cancer cells. Clin Cancer Res 2000;6:3739-47.

11. Bozec A, Sudaka A, Fischel JL, et al. Combined effects of bevacizumab with erlotinib and irradiation: a preclinical study on a head and neck cancer orthotopic model. Br J Cancer 2008;99:93-9.

12. Schicher N, Paulitschke V, Swoboda A, et al. Erlotinib and bevacizumab have synergistic activity against melanoma. Clin Cancer Res 2009;15:3495-502.

13. Herbst RS, Ansari R, Bustin F, et al. Efficacy of bevacizumab plus erlotinib versus erlotinib alone in advanced non-small-cell lung cancer after failure of standard first-line chemotherapy $(\mathrm{BeTa})$ : a double-blind, placebo-controlled, phase 3 trial. Lancet 2011;377:1846-54

14. Ciuleanu T, Tsai CM, Tsao CJ, et al. A phase II study of erlotinib in combination with bevacizumab versus chemotherapy plus bevacizumab in the first-line treatment of advanced non-squamous non-small cell lung cancer. Lung Cancer 2013;82:276-81.

15. Higgins J, Green S. Cochrane handbook for systematic reviews of interventions version 5.1.0 [updated March 2011]. The Cochrane collaboration, 2011. http://www.Cochrane-handbook.Org.

16. Guyatt $\mathrm{GH}$, Oxman AD, Vist GE, et al. GRADE: an emerging consensus on rating quality of evidence and strength of recommendations. BMJ 2008;336:924-6.

17. Cochran WG. The combination of estimates from different experiments. Biometrics 1954;10:101-29.

18. Higgins JP, Thompson SG, Deeks JJ, et al. Measuring inconsistency in meta-analyses. BMJ 2003;327:557-60.

19. DerSimonian R, Laird N. Meta-analysis in clinical trials. Control Clin Trials 1986;7:177-88.

20. Mantel N, Haenszel W. Statistical aspects of the analysis of data from retrospective studies of disease. J Natl Cancer Inst 1959;22:719-48.

21. Tierney JF, Stewart LA, Ghersi D, et al. Practical methods for incorporating summary time-to-event data into meta-analysis. Trials 2007;8:16.

22. Gridelli C, Bennouna J, de Castro J, et al. Randomized phase Illb trial evaluating the continuation of bevacizumab beyond disease progression in patients with advanced non-squamous non-small-cell lung cancer after first-line treatment with bevacizumab plus platinum-based chemotherapy: treatment rationale and protocol dynamics of the AvaALL (MO22097) trial. Clin Lung Cancer 2011;12:407-11.

23. Tanaka S, Sakamori $\mathrm{Y}$, Niimi M, et al. Design paper: a phase II study of bevacizumab and erlotinib in patients with non-squamous non-small cell lung cancer that is refractory or relapsed after 1-2 previous treatment (BEST). Trials 2011;12:120. 
24. Riggs $\mathrm{H}$, Jalal SI, Baghdadi TA, et al. Erlotinib and bevacizumab in newly diagnosed performance status 2 or elderly patients with nonsquamous non-small-cell lung cancer, a phase II study of the Hoosier Oncology Group: LUN04-77. Clin Lung Cancer 2013;14:224-9.

25. Zappa F, Droege C, Betticher D, et al. Bevacizumab and erlotinib (BE) first-line therapy in advanced non-squamous non-small-cell lung cancer (NSCLC) (stage IIIB/IV) followed by platinum-based chemotherapy (CT) at disease progression: a multicenter phase II trial (SAKK 19/05). Lung Cancer 2012;78:239-44.

26. Akerley W, Boucher K, Rich N, et al. A phase II study of bevacizumab and erlotinib as initial treatment for metastatic non-squamous, non-small cell lung cancer with serum proteomic evaluation. Lung Cancer 2013;79:307-11.

27. Cohen EE, Subramanian J, Gao F, et al. Targeted and cytotoxic therapy in coordinated sequence (TACTICS): erlotinib, bevacizumab and standard chemotherapy for non-small-cell lung cancer, a phase II trial. Clin Lung Cancer 2012;13:123-8.

28. Dingemans AM, de Langen AJ, van den Boogaart V, et al. First-line erlotinib and bevacizumab in patients with locally advanced and/or metastatic non-small-cell lung cancer: a phase II study including molecular imaging. Ann Oncol 2011;22:559-66.

29. Smith DA, Conkling P, Richards DA, et al. Antitumor activity and safety of combination therapy with the Toll-like receptor 9 agonist IMO-2055, erlotinib, and bevacizumab in advanced or metastatic non-small cell lung cancer patients who have progressed following chemotherapy. Cancer Immunol Immunother 2014;63:787-96.

30. Riely GJ, Rizvi NA, Kris MG, et al. Randomized phase II study of pulse erlotinib before or after carboplatin and paclitaxel in current or former smokers with advanced non-small-cell lung cancer. J Clin Oncol 2009;27:264-70

31. Carbone DP, Salmon JS, Billheimer D, et al. VeriStrat classifier for survival and time to progression in non-small cell lung cancer (NSCLC) patients treated with erlotinib and bevacizumab. Lung Cancer 2010;69:337-40.

32. Seto T, Kato T, Nishio M, et al. Erlotinib alone or with bevacizumab as first-line therapy in patients with advanced non-squamous non-small-cell lung cancer harbouring EGFR mutations (JO25567): an open-label, randomised, multicentre, phase 2 study. Lancet Oncol 2014;15:1236-44.

33. Herbst RS, O'Neill VJ, Fehrenbacher L, et al. Phase II study of efficacy and safety of bevacizumab in combination with chemotherapy or erlotinib compared with chemotherapy alone for treatment of recurrent or refractory non small-cell lung cancer. $J$ Clin Oncol 2007;25:4743-50.

34. Johnson BE, Kabbinavar F, Fehrenbacher L, et al. ATLAS: randomized, double-blind, placebo-controlled, phase IIIB trial comparing bevacizumab therapy with or without erlotinib, after completion of chemotherapy, with bevacizumab for first-line treatment of advanced non-small-cell lung cancer. J Clin Oncol 2013;31:3926-34.

35. Miller V, Soh $\mathrm{C}-\mathrm{H}$, Kabbinavar F. A randomized, double-blind, placebo-controlled, phase Illb trial (ATLAS) comparing bevacizumab (B) therapy with or without erlotinib (E) after completion of chemotherapy with B for first-line treatment of locally advanced, recurrent, or metastatic non-small cell lung cancer (NSCLC). J Clin Oncol 2009;27(18s):(abstr LBA8002).

36. Kabbinavar $\mathrm{F}$, Johnson BE, O'Connor $\mathrm{P}$, et al. Overall survival (OS) in ATLAS, a phase IIlb trial comparing bevacizumab (B) therapy with or without erlotinib (E) after completion of chemotherapy (chemo) with B for first-line treatment of locally advanced, recurrent, or metastatic non-small cell lung cancer (NSCLC). J Clin Oncol 2010;28(15S):(abstr 7526).

37. Sheng M, Zhao Y, Wang F, et al. Targeted drugs for unselected patients with advanced non-small-cell lung cancer: a network meta-analysis. J Thorac Dis 2016;8:98-115.

38. Falchook GS, Naing A, Hong DS, et al. Dual EGFR inhibition in combination with anti-VEGF treatment: a phase I clinical trial in non-small cell lung cancer. Oncotarget 2013;4:118-27.

39. Herbst RS, Johnson DH, Mininberg E, et al. Phase I/II trial evaluating the anti-vascular endothelial growth factor monoclonal antibody bevacizumab in combination with the HER-1/epidermal growth factor receptor tyrosine kinase inhibitor erlotinib for patients with recurrent non-small-cell lung cancer. J Clin Oncol 2005;23:2544-55.

40. Tortora G, Ciardiello F, Gasparini G. Combined targeting of EGFR-dependent and VEGF-dependent pathways: rationale, preclinical studies and clinical applications. Nat Clin Pract Oncol 2008:5:521-30.

41. Brugger $\mathrm{W}$, Triller N, Blasinska-Morawiec M, et al. Prospective molecular marker analyses of EGFR and KRAS from a randomized, placebo-controlled study of erlotinib maintenance therapy in advanced non-small-cell lung cancer. J Clin Oncol 2011;29:4113-20.

42. Cappuzzo F, Ciuleanu T, Stelmakh L, et al. Erlotinib as maintenance treatment in advanced non-small-cell lung cancer: a multicentre, randomised, placebo-controlled phase 3 study. Lancet Oncol 2010;11:521-9. 